\title{
Generalized Measures of Discrimination between Past Life Time Distributions
}

\author{
D.S. Hooda \\ Jaypee University of Engineering and Technology \\ Guna, India \\ ds_hooda@rediffmail.com \\ Sonali Saxena \\ Jaypee University of Engineering and Technology \\ Guna, India \\ sonalisaxena_2007@rediffmail.com
}

\begin{abstract}
In the present paper we define a generalized measure of discrimination between two past lifetime distributions of a system. We also characterize a propotional reversed hazard model and study its important properties.
\end{abstract}

\section{Introduction}

Ebrahimi and Pellerey (1995) and Ebrahimi (1996) have opined that Shannon's Information measure is a suitable tool to measure the uncertainty related to random lifetime distributions and their reliabilities.

Let $\mathrm{X}$ and $\mathrm{Y}$ be absolutely continuous non-negative honest random variables that describe the lifetime of two items. We denote by $\mathrm{f}(\mathrm{t}), \mathrm{F}(\mathrm{t})$ and $\bar{F}(t)=1-\mathrm{F}(\mathrm{t})$ the probability density function (p.d.f.), the cumulative distribution function (c.d.f.), and the survival function of $X$, respectively. Further we denote $\mathrm{g}(\mathrm{t}), \mathrm{G}(\mathrm{t})$ and $\bar{G}(t)$ the corresponding functions of Y. Moreover, Let $\lambda_{X}(x)=f(x) / \bar{F}(x)$ and $\lambda_{y}(x)=g(x) / \bar{G}(x)$ be the hazard rate functions of $\mathrm{X}$ and $\mathrm{Y}$, respectively, whereas $\tau_{X}(x)=f(x) / F(x)$ and $\tau_{Y}(x)=g(x) / G(x)$ will denote their reversed hazard rate functions. Here we may assume that densities $f(t)$ and $g(t)$ have support $(0,+\infty)$.

As an information distance between $F$ and $G$, Kullback and Leibler (1951) proposed the following discrimination measure, also known as relative entropy of $\mathrm{X}$ and $\mathrm{Y}$.

$$
I_{X, Y}(t)=\int_{0}^{+\infty} f(x) \log f(x) / g(x) d x,
$$

where 'log' denotes natural logarithm. Distance (1.1) is shift and scale invariant. It is also known as the relative entropy, or the directed information distance between $X$ and Y. Kullback (1959) strongly propogated its use in hypothesis testing and model evaluation. Since then it has been widely used in statistics and economics. 
Furthermore, $I_{X, Y} \geq 0$ is not 'a metric', as it does not satisfy the triangle inequality and is asymmetric. A symmetrized version of $I_{X, Y}$ introduced in Kullback and Leibler (1951) is defined by

$$
\begin{aligned}
\hat{I}_{X, Y} & =I_{X, Y}+I_{Y, X} \\
& =\int_{0}^{\infty}(f(x)-g(x)) \log \frac{f(x)}{g(x)} d x .
\end{aligned}
$$

In survival analysis and in life testing the current age of the systems under consideration is known and so it is taken in to account when two systems are compared this can be done by replacing $X$ and $Y$ by the distributions of corresponding residual lifetimes.

Accordingly, Ebrahimi and Kirmani (1996a) have defined the Kullback -Leibler discrimination information of $\mathrm{X}$ and $\mathrm{Y}$ at time $\mathrm{t}$ by

$$
I_{X, Y}(t)=\int_{t}^{+\infty} \frac{f(x)}{\bar{F}(t)} \log \frac{f(x) / \bar{F}(x)}{g(x) / \bar{G}(x)} d(x), \mathrm{t}>0
$$

Thus, analogous to (1.1), $I_{X, Y}(t)$ identifies with the relative entropy of $[X-t \mid X>t]$ and $[Y-t \mid Y>t]$. Information measure (1.3) is useful to compare the residual lifetimes of two items that have both survival up to time $t$.

Crescenzo and Longobardi (2004) have defined another information measure $\bar{I}_{X, Y}(t)$ dual to (1.3) in the sense that it is an information distance between the past lives $[X \mid X \leq t]$ and $[Y \mid Y \leq t]$. It is defined as follows

$$
\bar{I}_{X, Y}(t)=\int_{0}^{t} \frac{f(x)}{F(t)} \log \frac{f(x) / F(t)}{g(x) / G(t)} d x, \mathrm{t}>0 .
$$

They called (1.4) as measure of discrimination between past life time distributions.

Given that at time t, two items have been found to be failing, $\bar{I}_{X, Y}(t)$ measures the discrepancy between their past lives, where $\bar{I}_{X, Y}(t) \geq 0$. From (1.4), we have

$$
\bar{I}_{X, Y}(t)=-\int_{0}^{t} \frac{f(x)}{F(t)} \log \frac{g(x)}{G(t)}-\bar{H}_{X}(t),
$$

where $\bar{H}_{X}(t)=-\int_{0}^{t} \frac{f(x)}{F(t)} \log \frac{f(x)}{F(t)} d x$. 
We can write (1.4) in another way as given below:

$$
\bar{I}_{X, Y}(t)=\log \frac{G(t)}{F(t)}+\frac{1}{F(t)} \int_{0}^{t} f(x) \log \frac{f(x)}{g(x)} d x, t>0 .
$$

The measure (1.4) can be generalized in many ways. Here we study parametric generalization by introducing a parameter $\beta$ in section 2 . Thus we get a class of discrimination measures of which (1.4) is a particular case. New generalized measure has more flexibility in application due to infinite values of $\beta$. This has illustrated in section 3 by ploting graphs for different values of $\beta$. In section 4 we have studied characterization of proportional reversed hazard model.

\section{A Generalized Measure of Discrimination}

As we have mentioned earlier that measure (1.4) can be generalized in a number of ways. However, here we consider the following generalization

$$
\bar{I}^{\beta}{ }_{X, Y}(t)=\frac{1}{\beta-1}\left[\int_{0}^{t}\left(\frac{f(x)}{F(t)}\right)^{\beta} \cdot\left(\frac{g(x)}{G(t)}\right)^{1-\beta} d x-1\right], \beta \neq 1, \beta>0
$$

It may be noted that (2.1) reduces to (1.4), when $\beta \rightarrow 1$. So we may call (2.1) the discrimination information measure of degree $\beta$.

Consequently, the measure (1.5) can also be generalized as follows:

$$
\bar{I}_{X, Y}^{\beta}(t)=-\int_{0}^{t} \frac{f(x)}{F(t)}\left(\frac{g(x) / G(t)}{f(x) / F(t)}\right)^{1-\beta} \log \frac{g(x)}{G(t)} d x-\bar{H}_{X}^{\beta}(t),
$$

where $\bar{H}_{X}^{\beta}(t)=-\int_{0}^{t} \frac{f(x)}{F(t)}\left(\frac{g(x) / G(t)}{f(x) / F(t)}\right)^{1-\beta} \log \frac{f(x)}{F(t)} d x$.

Next we obtain some bounds of $\bar{I}_{\mathrm{X}, \mathrm{Y}}^{\beta}(t)$ using the term "increasing" and "decreasing" in non-strict sense. We also make use of the notions of stochastic orders and refer to Shaked and Shanthikumar (1994) for definition and basic results. As particular cases our results reduce to the results due to Crescenzo and Longobardi (2003).

Theorem 2.1 If $\frac{f(t)}{g(t)}$ is increasing in $\mathrm{t}>0$, i.e. $X \geq_{l r} Y$, then

$$
\bar{I}_{X, Y}^{\beta}(t) \leq\left(\frac{\tau_{X}(t)}{\tau_{Y}(t)}\right)^{\beta-1} \log \frac{\tau_{X}(t)}{\tau_{Y}(t)}
$$


Proof: $\quad$ From the measure (2.1), we have

$$
\begin{aligned}
\bar{I}_{X, Y}^{\beta}(t) & =\frac{1}{\beta-1}\left[\int_{0}^{t}\left(\frac{f(x)}{F(t)}\right)^{\beta} \cdot\left(\frac{g(x)}{G(t)}\right)^{1-\beta} d x-1\right] \\
& =\frac{1}{\beta-1}\left[\int_{0}^{t}\left(\frac{f(x)}{F(t)}\right) \cdot\left(\frac{G(t)}{g(x)}\right)^{\beta-1} \cdot\left(\frac{f(x)}{F(t)}\right)^{\beta-1} d x-1\right]
\end{aligned}
$$

Due to increasing of $\frac{f(t)}{g(t)}$, from (1.4) for all $\mathrm{t}>0$ we have

$$
\begin{aligned}
&=\frac{1}{\beta-1}\left[\int_{0}^{t}\left(\frac{f(x)}{F(t)}\right) \cdot\left(\frac{G(t)}{g(x)}\right)^{\beta-1} \cdot\left(\frac{f(x)}{F(t)}\right)^{\beta-1} d x-1\right] \\
& \leq \frac{1}{\beta-1}\left[\frac{1}{F(t)} \int_{0}^{t} f(x)\left(\frac{f(t) / F(t)}{g(t) / G(t)}\right)^{\beta-1} d x-1\right] \\
&= \frac{1}{F(t)} \int_{0}^{t} f(x) \cdot\left(\frac{f(t) / F(t)}{g(t) / G(t)}\right) \log \left(\frac{f(t) / F(t)}{g(t) / G(t)}\right) d x \\
&= \frac{1}{F(t)}\left(\frac{f(t) / F(t)}{g(t) / G(t)}\right)^{\beta-1} \log \left(\frac{f(t) / F(t)}{g(t) / G(t)}\right) \int_{0}^{t} f(x) d x . \\
&=\left(\frac{f(t) / F(t)}{g(t) / G(t)}\right)^{\beta-1} \log \left(\frac{f(t) / F(t)}{g(t) / G(t)}\right) \\
& \bar{I}_{X, Y}^{\beta}(t)=\left(\frac{\tau_{Y}(t)}{\tau_{Y}(t)}\right)^{\beta-1} \log \frac{\tau_{x}(t)}{\tau_{Y}(t)} . \text { Hence Theorem 2.1 is proved. }
\end{aligned}
$$

When $\frac{f(t)}{F(t)}$ is decreasing the proof is similar.

Again from (2.1), we have

$$
\begin{aligned}
& \bar{I}_{X, Y}^{\beta}(t)=\frac{1}{\beta-1}\left[\int_{0}^{t}\left(\frac{f(x)}{F(t)}\right)^{\beta}\left(\frac{g(x)}{G(t)}\right)^{\beta-1} d x-1\right] \\
&=\int_{0}^{t}\left(\frac{f(x)}{F(t)}\right) \cdot\left(\frac{g(x) / G(t)}{f(x) / F(t)}\right)^{1-\beta} \log \left(\frac{f(x) / F(t)}{g(x) / G(t)}\right) d x \\
&=\int_{0}^{t}\left(\frac{f(x)}{F(t)}\right) \cdot\left(\frac{g(x) / G(t)}{f(x) / F(t)}\right)^{1-\beta} \log \left(\frac{g(x)}{G(t)}\right) d x-\bar{H}_{X}^{\beta}(t), \\
& \text { where } \bar{H}_{X}^{\beta}(t)=\int_{0}^{t} \frac{f(x)}{F(t)}\left(\frac{g(x) / G(t)}{f(x) / F(t)}\right)^{\beta-1} \log \frac{f(x)}{F(t)} .
\end{aligned}
$$


If $g(x) \geq g(t)$ for all $t>x>0$, then

$$
\begin{aligned}
& \int_{0}^{t}\left(\frac{f(x)}{F(x)}\right) \cdot\left(\frac{g(x) / G(t)}{f(x) / F(t)}\right)^{1-\beta} \log \left(\frac{g(x)}{G(t)}\right) d x-\bar{H}_{X}^{\beta}(t) \leq \int_{0}^{t}\left(\frac{f(x)}{F(t)}\right) \cdot\left(\frac{f(t) / F(t)}{g(t) / G(t)}\right)^{\beta-1} \log \frac{g(t)}{G(t)} d x-\bar{H}_{X}^{\beta}(t) . \\
& =\left(\frac{f(t) / F(t)}{g(t) / G(t)}\right)^{\beta-1} \log \frac{g(t)}{G(t)}-\bar{H}_{X}^{\beta}(t) \\
& =\left(\frac{\tau_{X}(t)}{\tau_{Y}(t)}\right)^{\beta-1} \log \frac{g(t)}{G(t)}-\bar{H}_{X}^{\beta}(t), t>0
\end{aligned}
$$

\section{Applications}

Let $X$ and $Y$ have weighted exponential distributions with rates $\lambda$ and $\mu$ respectively and with weight function $\phi(t)$. Their densities are given by

$$
f(t)=\frac{\phi(t) \cdot e^{-\lambda t}}{h(\lambda)}, \quad g(t)=\frac{\phi(t) e^{-\mu t}}{h(\mu)}, t>0 .
$$

where $\mathrm{h}($.$) denotes the Laplace transform of \phi(t)$ :

$$
h(\xi)=L \xi[\phi(t)]=\int_{0}^{\infty} e^{-\xi t} \phi(t) d t, \quad \xi>0 .
$$

Solving the measure (1.6) and (3.1), Crescenzo and Longobadi expressed the discrimination measure of the past lives for $\lambda \neq \mu$ as follows

$$
\bar{I}_{X, Y}(t)=\log \frac{G(t)}{F(t)}+\log \frac{h(\mu)}{h(\lambda)}+(\mu-\lambda) E[X \mid X \leq t], \quad t>0 .
$$

Next we generalize measure (3.2) in the following way:

$$
\begin{aligned}
\bar{I}_{X, Y}^{\beta}(t) & =\frac{1}{F(t)}\left[\left(\frac{G(t)}{F(t)}\right)^{\beta-1} \log \left(\frac{G(t)}{F(t)}\right) \int_{0}^{t} f(x) \cdot\left(e^{(\mu-\lambda) x} \cdot \frac{h(\mu)}{h(\lambda)}\right)^{\beta} d x\right] \\
& +\left(\frac{h(\mu)}{h(\lambda)}\right)^{\beta-1}(\mu-\lambda)\left(\frac{G(t)}{F(t)}\right)^{\beta-1} \int_{0}^{t} x f(x)\left(e^{(\mu-\lambda) x}\right)^{\beta-1} d x \\
& +\left(\frac{G(t)}{F(t)}\right)^{\beta-1} \cdot \log \frac{h(\mu)}{h(\lambda)} \cdot\left(\frac{h(\mu)}{h(\lambda)}\right)^{\beta-1} \int_{0}^{t} f(x) \cdot\left(e^{(\mu-\lambda)}\right)^{\beta-1} d x
\end{aligned}
$$

It may be noted that (3.3) reduces to (3.2) when $\beta \rightarrow 1$.

Example 3.1 Let $X$ and $Y$ be Erlang distributed, with scale parameter $\lambda$ and $\mu$ respectively, with the common shape parameter $\mathrm{n}$. Hence they posses densities given by (3.1), where $\phi(t)=t^{n-1}$. and $h(\xi)=(n-1) ! \xi^{n}$. 
If we put $f(x)=\frac{\phi(x) \cdot e^{-\lambda x}}{h(\lambda)}, \quad$ where $h(\lambda)=\frac{(n-1) !}{\lambda^{n}} \quad$ and $\phi(x)=x^{n-1}$ in (3.3),

then from (3.3), we have

$$
\begin{aligned}
\bar{I}_{X, Y}^{\beta}(t) & =\frac{1}{F(t)}\left[\left(\frac{G(t)}{F(t)}\right)^{\beta-1} \log \left(\frac{G(t)}{F(t)}\right) \int_{0}^{t} f(x) \cdot\left(e^{(\mu-\lambda) x} \cdot \frac{h(\mu)}{h(\lambda)}\right)^{\beta-1} d x\right. \\
& +\left(\frac{h(\mu)}{h(\lambda)}\right)^{\beta-1}(\mu-\lambda) \cdot\left(\frac{G(t)}{F(t)}\right)^{\beta-1} \int_{0}^{t}\left(x \cdot e^{-\lambda x} x^{n-1}\right) \lambda^{n} d x \\
& \left.+\left(\frac{G(t)}{F(t)}\right)^{\beta-1} \cdot n \log \frac{\lambda}{\mu}\left(\frac{h(\mu)}{h(\lambda)}\right)^{\beta-1} \int_{0}^{t} f(x) \cdot\left(e^{(\mu-\lambda) x}\right) d x\right] .
\end{aligned}
$$

In case $\beta \rightarrow 1,(3.4)$ reduces to $\bar{I}_{(X, Y)}(t)=\log \frac{G(t)}{F(t)}+\frac{(\mu-\lambda)}{F(t)} \cdot \frac{\gamma((n+1), \lambda t)}{(n-1) ! \cdot \lambda}+n \log \frac{\lambda}{\mu}$.

This is clearly indicated in the Figure 3.1 and 3.2

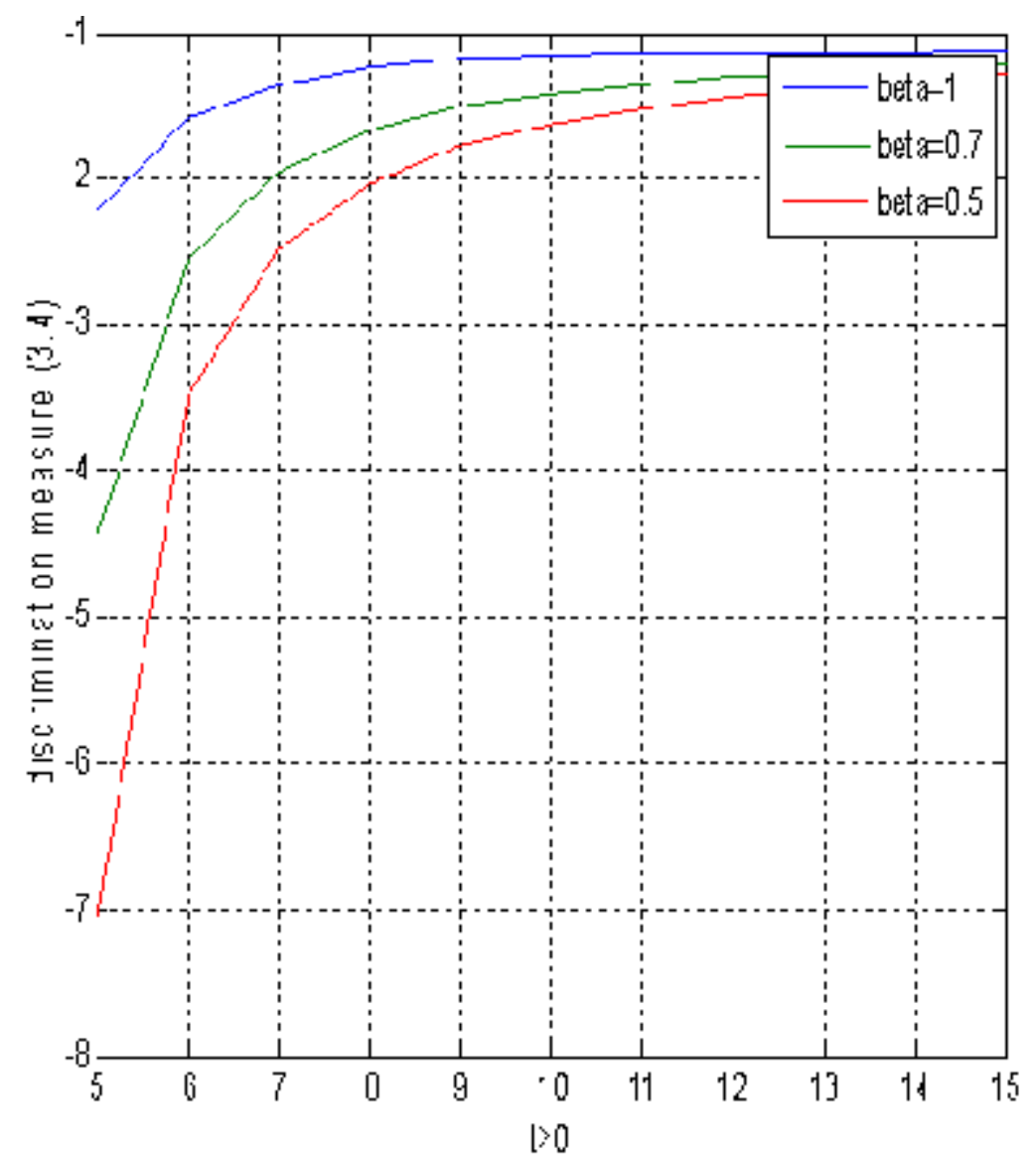

Figure 3.1: The discrimination measure (3.4) for $\mu>\lambda$ (from bottom to top) 


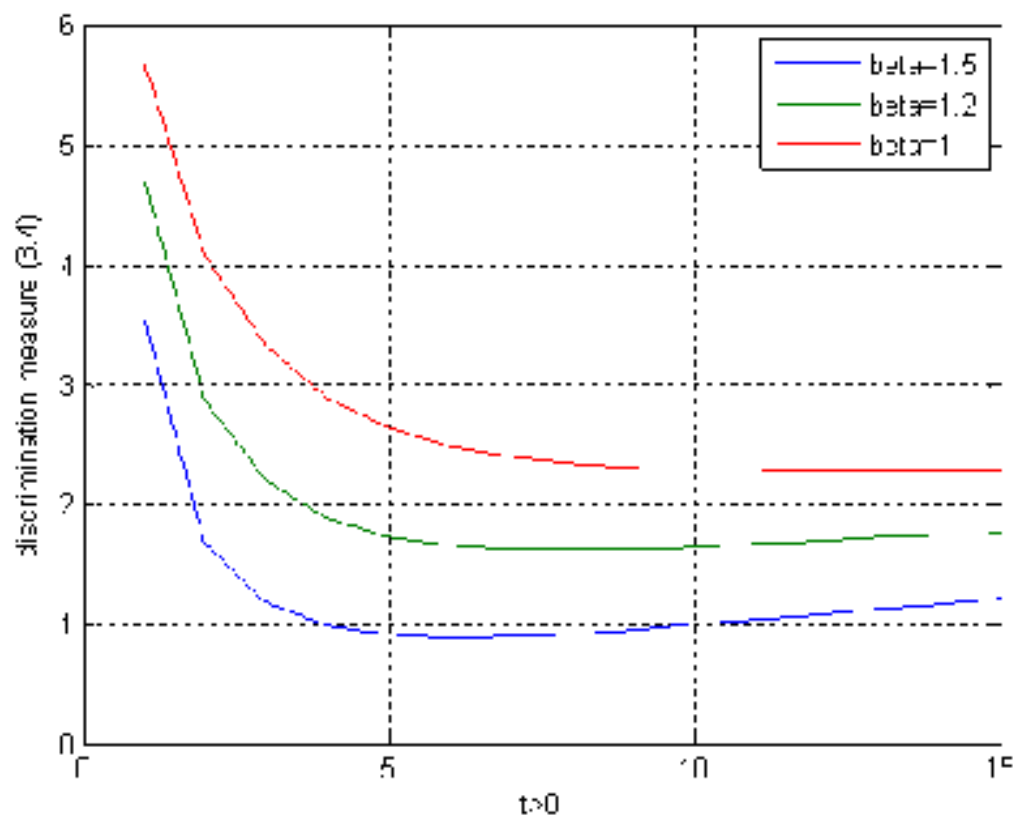

Figure 3.2: The discrimination measure (3.4) for $\mu<\lambda$

The following example shows that discrimination measure $\bar{I}_{X, Y}^{\beta}(t)$ is not necessarily monotone.

\section{Example 3.2 Let}

$$
\begin{aligned}
& F(x)= \begin{cases}\exp \{-1 / 2-1 / x\} & \text { if } 0<x \leq 1, \\
\exp \left\{-2+x^{2} / 2\right\} & \text { if } 1<x \leq 2,\end{cases} \\
& G(x)= \begin{cases}x^{2} / 4 & \text { if } 0<x \leq 2, \\
1 & \text { if } x \geq 2\end{cases}
\end{aligned}
$$

be the c.d.f.'s of $X$ and $Y$. From Figure 3.3 we see that $\bar{I}_{X, Y}^{\beta}(t)$ is not monotone for all $t \in(0,2)$. 


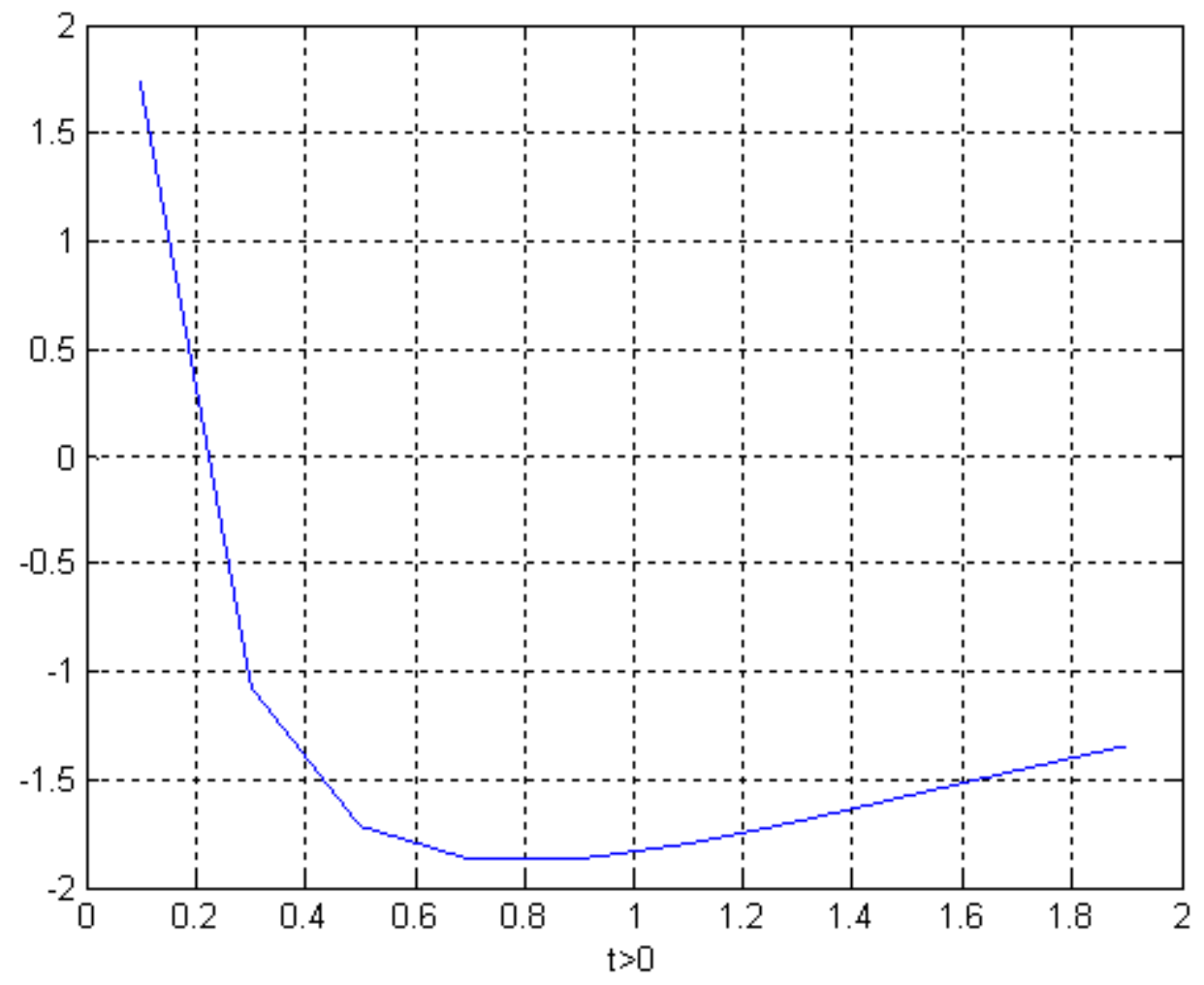

Figure 3.3 The discrimination measure of past lives of example (3.2) is plotted for $\mathrm{t}>0$

\section{Characterization of Proportional Reversed Hazards Model}

Let $X$ be a random life time i.e. a non-negative absolutely continuous random variable with probability density function $f(x)$, cumulative distribution function $F(x)$ and survival function $\bar{F}(x)=1-F(x)$.Suppose that $(0, b)$ is the interval of support of $\mathrm{F}(\mathrm{x})$, with $0<b<\infty$. Then we have following definition:

Definition 4.1: Hazard rate function of $X$

$$
\begin{aligned}
& \lambda_{X}(x)=-\frac{d}{d x} \log \bar{F}(x)=\frac{f(x)}{F(x)}, \quad x<b, \text { is hazard rate function of } X \text { and } \\
& \tau_{X}(x)=\frac{d}{d x} \log F(x)=\frac{f(x)}{F(x)}, x>0 .
\end{aligned}
$$

is reversed hazard rate function of $X$.

The proportional reversed hazard rate model is expressed by a non-negative absolutely continuous random variable $Y$ whose distribution is the power of the distribution function of $\mathrm{X}$ as given below:

$$
G(x)=(F(x))^{\theta}, x \in R,
$$


where $\theta$ is a positive real number. Probability density and hazard rate functions of $Y$ are, respectively,

$$
\begin{aligned}
& g_{Y}(x)=\frac{d}{d x} G(x)=\theta[F(x)]^{\theta-1} f(x), x \in R \\
& \lambda_{Y}(x)=\frac{g(x)}{\bar{G}(x)}=\theta \lambda_{Y}(x) \cdot \xi(x), x<b .
\end{aligned}
$$

Where

$$
\xi(x)=\frac{(F(x))^{\theta-1}-(F(x))^{\theta}}{1-(F(x))^{\theta}}
$$

This function has a probabilistic interpretation when $\theta$ is a positive integer. From (4.1) and (4.2(a)) it follows that the reversed hazard rate function of $Y$ is given by $\tau_{Y}(x)=\frac{g(x)}{G(x)}=\theta \cdot \tau_{X}(x), x>0$.

Example 4.1: Consider a parallel system of $\mathrm{n}$ components having i.i.d. life time $Y_{i}, i=1,2, \ldots \ldots \ldots . . . n$.characterized by the p.d.f. $g(x)$ and c.d.f. $G(x)$. The lifetime of the system is then $X=\max \left\{Y_{1}, Y_{2}, \ldots \ldots \ldots \ldots \ldots, Y_{n}\right\}$, with p.d.f. $f(x)=n G^{n-1}(x) g(x)$ and c.d.f. $F(x)=G^{n}(x)$. Hence $X$ and $Y_{i}$ satisfy the proportional reversed hazard model.

From measure (2.2), for $t>0$ and $i=1,2,3, \ldots \ldots \ldots \ldots \ldots$, we have

$$
\bar{I}_{X, Y}^{\beta}(t)=\frac{1}{\beta-1}\left[\int_{0}^{t} \frac{f(x)}{F(t)} \cdot\left(\frac{g(x) / G(t)}{f(x) / F(t)}\right)^{1-\beta} d x-1\right] .
$$

Solving above equation we have

$$
\begin{aligned}
\bar{I}_{X, Y}^{\beta}(t) & =\frac{1}{\beta-1}\left\{\frac{(n)^{\beta} \cdot\left(G^{n-1}(t)\right)^{1-\beta}}{G^{n}(t)} \cdot \frac{G(t)^{n \beta-\beta+1}}{n \beta-\beta+1}-1\right\} \\
= & \frac{1}{\beta-1}\left\{(n)^{\beta} \cdot \frac{\left(G^{n-1}(t)\right)^{1-\beta}}{G^{n}(t)} \cdot \frac{G(t)^{n \beta-\beta+1}}{n \beta-\beta+1}-1\right\} \\
& =\frac{1}{\beta-1}\left\{(n)^{\beta} \cdot \frac{G^{(n-1)(1-\beta)}(t)}{G^{n}(t)} \cdot \frac{G^{(n \beta-\beta+1)}(t)}{n \beta-\beta+1}-1\right\} \\
& =\frac{1}{\beta-1}\left\{(n)^{\beta} \cdot(n \beta-\beta+1)^{-1}-1\right\} \\
\bar{I}_{X, Y)}^{\beta}(t) & =n^{\beta} \log n \cdot(n \beta-\beta+1)^{-1}-(n)^{\beta} \cdot(n \beta-\beta+1)^{-2} \cdot(n-1)
\end{aligned}
$$


For $\beta \rightarrow 1,(4.3)$ reduces to

$$
\bar{I}_{X, Y}(t)=\log n-\frac{(n-1)}{n} \text {. }
$$

Example 4.2: Let us consider a parallel system of $\mathrm{n}$ components characterized by i.i.d. lifetime $X_{i}, i=1,2,3, \ldots \ldots \ldots \ldots \ldots . . n$ and let $Y=\max \left\{X_{1}, X_{2}, \ldots \ldots \ldots \ldots \ldots, X_{n}\right\}$ be the lifetime of the p.d.f. of $X_{i}$. The c.d.f. of $\mathrm{Y}$ is $G(x)=F^{n}(x)$ and its $g(x)=n F^{n-1}(x) \cdot f(x)$.

Thus we have

$$
\begin{aligned}
\bar{I}_{X, Y}^{\beta}(t) & =\frac{1}{\beta-1}\left[\int_{0}^{t}\left(\frac{f(x)}{F(t)}\right)^{\beta} \cdot\left(\frac{g(x)}{G(t)}\right)^{1-\beta} d x-1\right] \\
& =\frac{1}{\beta-1}\left[\int_{0}^{t}\left(\frac{f(x)}{F(t)}\right)^{\beta} \cdot\left(\frac{n F^{n-1}(x) \cdot f(x)}{F^{n}(t)}\right)^{1-\beta} d x-1\right] \\
& =\frac{1}{\beta-1}\left[\frac{n^{1-\beta}}{F(t) \cdot F^{(n-1)(1-\beta)}(t)} \int_{0}^{t} F^{(n-1)(1-\beta)}(x) \cdot F^{(n-1(1-\beta)} \cdot F^{\prime}(x) d x-1\right] \\
& =\frac{1}{\beta-1}\left\{\frac{n^{1-\beta}}{n-(n-1) \beta}-1\right\} \\
& =\frac{1}{\beta-1}\left\{n^{1-\beta}(n-(n-1) \beta)^{-1}-1\right\} \\
\bar{I}_{X, Y}^{\beta}(t) & =\left\{n^{1-\beta} \log n(n-(n-1) \beta)^{-1}+n^{1-\beta}[n-(n-1) \beta]^{-2}(n-1)\right\}
\end{aligned}
$$

For $\beta \rightarrow 1(4.5)$ reduces to

$$
\bar{I}_{X, Y}(t)=-\log n+(n-1)=n-1-\log n .
$$

It may be noted that if we let $n=\frac{1}{m}$ in (4.6), we get (4.4).this proves that the relevant connection between $X$ and Yare proportional with factor $n$, while this property is not satisfied by their hazard rate functions except $n=1$.

\section{Conclusion}

The directed divergence or discrimination measure or relative entropy has an analogus with residual life in reliability theory. In the present paper we have studied a generalized discrimination measure of past life distribution of a system 
at time $\mathrm{t}$ and have characterized a proportional reversed hazard model. This study has a connection with Residual failure rate in survival analysis.

\section{References}

1. Di Crescenzo, A. and Longobardi, M. (2004). A Measure of Discrimination between past lifetime distributions. Probab. Letters. 67, 173-182.

2. Di Crescenzo, A. (2000). Some results on the proportional reversed hazards model. Statist. Probab. Lett, 50, 313-321.

3. Di Crescenzo, A. Longobardi, M., (2002). Entropy-based measure of uncertainty in past lifetime distributions. J. Appl. Prob. 39, 434-440.

4. Ebrahimi, N. (1996). How to measure uncertainty in the residual life time distribution. Sankhya Ser. A. 58 48-56.

5. Ebrahimi N. Kirmani, S.N.U.A. (1996a). A measure of discrimination between two residual life time distributions and its applications. Ann. Inst. Statist. Math. 48, 257-265.

6. Ebrahimi, N., Pellerey, F. (1995). New partial ordering of survival functions based on the notion of uncertainty. J. Appl. Prob. 32, 202-211.

7. Kullback, S. Leibler, R.A. (1951). On information and sufficiency. Ann. Math. Statist. 22, 79-86.

8. Kullback S. (1959). Information Theory and Statistics. New York: John Wiley.

9. Shaked M. Shantikumar, J.G. (1994). Stochastic Orders and Their applications. Acadimic Press, San Diego. 